\title{
Exploring Anti-Klebsiella pneumoniae Activity of Probiotic Lactobacilli of Curd Origin
}

\section{Shyamapada Mandal* and Debashis Halder}

Laboratory of Microbiology and Experimental Medicine, Department of Zoology, University of Gour Banga, Malda, India

*Corresponding Author: Shyamapada Mandal, Profeesor, Laboratory of Microbiology and Experimental Medicine, Department of Zoology, University of Gour Banga, Malda, West Bengal, India. E-mail: samtropmed@gmail.com

Received: February 09, 2018; Published: March 21, 2018

DOI: $10.31080 / A S M I .2018 .01 .0038$

\begin{abstract}
The current research evaluates the bacterial growth inhibitory activity of indigenous probiotic lactobacilli and standard Lactobacillus strains against multidrug resistant (MDR) Klebsiella pneumoniae. The probiotic lactobacilli strains (Lactobacillus animalis LMEM6, L. plantarum LMEM7, L. acidophilus LMEM8 and L. rhamnosus LMEM9) from curd samples, and Lactobacillus fermentum MTCC 9748 standard strain were tested against indicator bacterial pathogens (n = 2): K. pneumoniae B535 clinical and K. pneumoniae NTCC 703603 standard strains, following agar overlay and agar-well diffusion techniques. The indicator bacterial strains were tested as MDR against 6 or more antibiotics with MAR (multiple antibiotic index) $0.4-0.73$. The ZDI (zone diameter of inhibition) values from the action of test lactobacilli strains ranged $13.00 \pm 1.00-33.33 \pm 1.53$, following agar-overlay method, and $12.00 \pm 1.00-22.00$ $\pm 1.00 \mathrm{~mm}$, following agar-well method, against MDR $K$. pneumoniae indicator strains. The "R" value of lactobacilli strains ranged $3.00-13.17 \mathrm{~mm}$, while the bacteriocin activity, in terms of activity unit (AU/ml), ranged $155.60-293.33$. The study suggests that the indigenous lactobacilli might play an important role in the protection of host against MDR K. pneumoniae infection, and such probiotic strains may beneficially be employed as biotherapeutic agents in partial replacement or adjunct to antibiotic therapy in the treatment of MDR K. pneumoniae infection.
\end{abstract}

Keywords: Probiotic Lactobacilli; MDR Klebsiella pneumoniae; Antagonistic Activity; Bacteriocin Activity; MAR Index

\section{Introduction}

The findings of beneficial bacteria, viz., probiotic lactobacilli, as preventive and therapeutic agents to eliminate potential bacterial pathogens are increasing globally [1,2]. The need of such new treatment is important in the treatment of health care-associated infections caused by antibiotic resistant (MDR: multidrug resistant; XDR: extensively drug-resistant; PDR: pandrug resistant) bacteria. The Klebsiella pneumoniae is such an opportunistic gram-negative bacterial pathogen, and has been recently recognized as a significant threat to global public health [3]. They are pervasive in nature and generally colonize a diversity of body sites including the human gut, and remain embedded forming biofilm that protects them from the action of antibiotics, and make the situation more difficult to treat the infection [4]. The lactobacilli (genus Lactobacillus), which are generally recognize as safe (GRAS), are the prime members of the intestinal microbiota of humans, and had already been reported as alternative to antibiotics as a promising candidate to compete with the harmful bacteria [2].
The combined antibacterial activity of lactobacilli strains (between L. animalis and L. gasseri) were reported and found effective against K. pneumoniae [5]. The lactobacilli have extensively been studied for their remarkable capacity to inhibit the growth of other microorganisms through the inhibitory property $[5,6]$. The antibiofilm activity of L. plantarum strain against $K$. pneumoniae has been reported by Lagrafeuille., et al [7]. The culture filtrates of lactobacilli strains were also reported as the inhibitory agent against K. pneumoniae [8]. However, no scientific report has been recorded on the prevention of MDR $K$. pneumoniae strain with probiotic therapy from indigenous lactobacilli strains, at least from this part of the globe. Hence, the present study has been aimed to explore the antagonistic activity of probiotic curd lactobacilli strains as alternative therapeutics to prevent MDR K. pneumoniae infection.

\section{Materials and Methods}

Bacterial strains

Four curd lactobacilli probiotic strains: Lactobacillus animalis LMEM6, L. plantarum LMEM7, L. acidophilus LMEM8, L. rhamnosus LMEM9, were utilized in the current study. The methodol- 
ogy details for procurement and identification of the isolates were explained earlier [2]. The standard strain used was Lactobacillus fermentum MTCC 9748. All the strains were maintained in MRS broth (Hi-Media, India) culture and MRS agar (Hi-Media, India) stabs, in the Laboratory of Microbiology and Experimental Medicine, Department of Zoology, University of Gour Banga, Malda. The indicator strains used in the study included K. pneumoniae $(\mathrm{n}=2)$ : K. pneumoniae B535 clinical and K. pneumoniae NTCC 703603 standard strains, which were maintained in the same laboratory in nutrient broth (Hi-Media, India) culture as well as in cystine tryptone agar (Hi-Media, India) stabs.

\section{Antibiotic susceptibility of indicator bacteria}

The antibiotic susceptibility of the indicator bacterial strains $(K$ pneumoniae B535 clinical and K. pneumoniae NTCC 703603 standard) was determined by disc diffusion method [9], using MuellerHinton agar (Hi-Media, India), and 15 antibiotic discs (Hi-Media, India): Ak: amikacin (30- $\mu \mathrm{g} / \mathrm{disc}) ;$ Amc: amoxyclav $(30-\mu \mathrm{g} / \mathrm{disc})$; Amp: ampicillin (10- $\mu \mathrm{g} / \mathrm{disc})$; Cpd: cefpodoxime (10- $\mu \mathrm{g} / \mathrm{disc}) ; \mathrm{Cx}$ : cefoxitin $(30-\mu \mathrm{g} / \mathrm{disc})$; Ctx: cefotaxime $(30-\mu \mathrm{g} / \mathrm{disc}) ; \mathrm{Gen}$ : gentamicin $(10-\mu \mathrm{g} /$ disc); Ipm: imipenem $(10-\mu \mathrm{g} / \mathrm{disc}) ; \mathrm{K}$ : kanamycin (30- $\mu \mathrm{g} /$ disc); Met: methicillin (5- $\mu \mathrm{g} / \mathrm{disc}) ;$ Mrp: meropenem (10$\mu \mathrm{g} /$ disc); Te: tetracycline $(10-\mu \mathrm{g} /$ disc); Na: Nalidixic acid $(10-\mu \mathrm{g} /$ disc); Tr: trimethoprim (5- $\mu \mathrm{g} /$ disc) and Va: vancomycin $(30-\mu \mathrm{g} /$ disc). The susceptibility test results, in terms of ZDI (zone diameter of inhibition) values, were interpreted as following the criteria of Clinical Laboratory Standards Institute [9], to label the isolates as resistant, sensitive or intermediately susceptible. The MAR (multiple antibiotic resistance) indices were calculated following standard protocol as described before [10].

\section{Antagonistic activity of lactobacilli}

The antagonistic activity of four indigenous lactobacilli: $L$. animalis LMEM6, L. plantarum LMEM7, L. acidophilus LMEM8, L. rhamnosus LMEM9, and the standard L. fermentum MTCC 9748 strain was determined by agar-well diffusion as well as agar overlay methods, against $K$. pneumoniae strains ( $\mathrm{n}=2)$, one clinical and one standard, as mentioned above. The results (ZDI: zone diameter of inhibition) obtained from the earlier method was interpreted according to the criteria mentioned elsewhere [2], while the ZDIs values from the later method were interpreted following Shokryazdan et al. [11]: the ZDI > $20 \mathrm{~mm}, 10-20 \mathrm{~mm}$ and < $10 \mathrm{~mm}$ were considered as strong, intermediate and weak inhibitions, respectively.

\section{Determination of " $R$ " value of lactobacilli}

The "R" (width of clear zone) values were also calculated, following agar overlay method, as per the formula stated earlier [5]: $\mathrm{R}=\frac{(\mathrm{d} \text { Inhib }-\mathrm{d} \text { Spot) }}{2}$ (where, "d Inhib": the diameter of clear zone around the "d Spot"; and "d Spot": the diameter of spot form of lactobacilli grown on MRS agar plate). The scores of " $R$ " were interpreted following the criteria mentioned earlier [12,13]. All the tests were performed in triplicate, and the data were represented as mean \pm SD (standard deviation).
Determination of $\mathrm{AU} / \mathrm{mL}$ of cell free supernatant of lactobacilli

The antagonistic activity of the test lactobacilli strains in terms of arbitrary unit per $\mathrm{mL}(\mathrm{AU} / \mathrm{mL})$ was calculated (mean $\pm \mathrm{SD})$ as 'a measure of production of bioactive components' using the formula mentioned elsewhere [14]: $\mathrm{AU} / \mathrm{mL}=\frac{Z D I \times 100}{\text { volume taken in the well }(\mu L)}$ ; where ZDI denotes “zone diameter of inhibition”, following agarwell diffusion method.

\section{Results and Discussion}

The gram-negative enteric bacteria, $K$. pneumoniae possesses the capacity to cause community-acquired as well as healthcareassociated infections, such as pneumonia, bloodstream infection, surgical site infections, urinary tract infection, intra-abdominal infection, skin and soft tissue infection, liver abscess and meningitis $[15,16]$. In addition, the antibiotic resistance, among K. pneumoni$a e$, is an emerging global healthcare crisis [17], and of particular the emergence of MDR K. pneumoniae incites impediment in the antibiotic therapy (even with last resort carbapenems: imipenem and meropenem) by escalating the dissemination of resistant pathogens causing protracted infection time among infected individuals $[17,18]$. In the current study, the indicator bacterial pathogens were MDR (Figure 1 and Table 1); K. pneumoniae B535 clinical strain showed resistance to 11 and K. pneumoniae NTCC 703603 standard to 6 antibiotics tested, thus having MAR index of 0.73 and 0.4 , respectively.

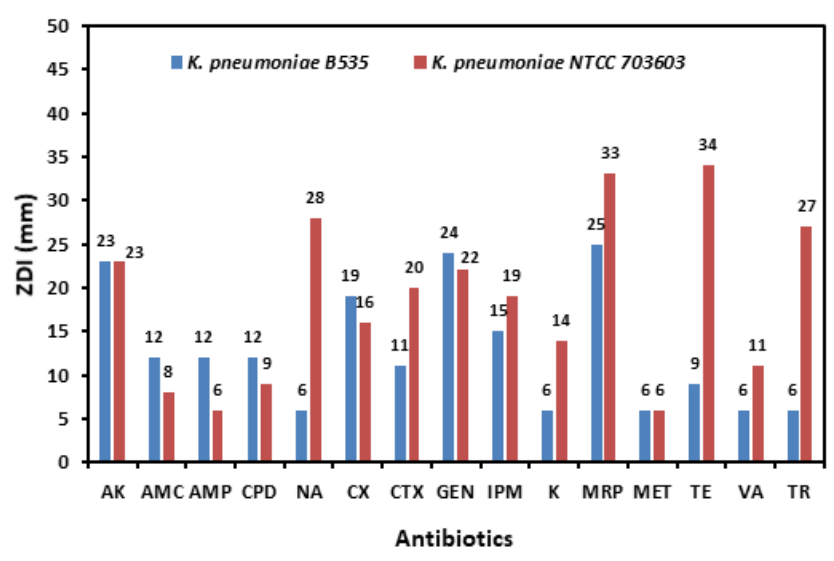

Figure 1: The ZDI (zone diameter of inhibition) values from antibiotic action against the indicator bacteria. Ak: Amikacin; Amc: Amoxyclav; Amp: Ampicillin; Cpd: Cefpodoxime; Cx: Cefoxitin; Ctx: Cefotaxime; Gen: Gentamicin; Ipm: Imipenem; K: Kanamycin; Met: Methicillin; Mrp: Meropenem; Te: Tetracycline; Na: Nalidixic Acid; Tr: Trimethoprim; Va: Vancomycin. 


\begin{tabular}{|c|c|c|c|}
\hline Strain & Resistant & $\begin{array}{l}\text { Intermediately } \\
\text { susceptible }\end{array}$ & Sensitive \\
\hline $\begin{array}{l}\text { K. pneumoniae } \\
\text { B535 }\end{array}$ & $\begin{array}{c}\text { Amc, Amp, } \\
\text { Ctx, Cpd, } \\
\text { Ipm, K, Met, } \\
\mathrm{Na}, \mathrm{Te}, \mathrm{Tr}, \mathrm{Va}\end{array}$ & - & $\begin{array}{c}\mathrm{Ak}, \mathrm{Cx}, \mathrm{Gen}, \\
\mathrm{Mrp},\end{array}$ \\
\hline $\begin{array}{l}\text { K. pneumoniae } \\
\text { NTCC } 703603\end{array}$ & $\begin{array}{c}\text { Amc, Amp, } \\
\text { Ctx, Cpd, } \\
\text { Met, Va }\end{array}$ & $\mathrm{Cx}, \mathrm{Ipm}, \mathrm{K}$ & $\begin{array}{c}\text { Ak, Gen, } \\
\text { Mrp, Na, } \\
\text { Te, Tr }\end{array}$ \\
\hline
\end{tabular}

Table 1: Antibiotic susceptibility test results for indicator bacteria.

Ak: Amikacin; Amc: Amoxyclav; Amp: Ampicillin; Cpd: Cefpodoxime; Cx: Cefoxitin; Ctx: Cefotaxime; Gen: Gentamicin; Ipm: Imipenem; K: Kanamycin; Met: Methicillin; Mrp: Meropenem; Te: Tetracycline; Na: Nalidixic Acid; Tr: Trimethoprim; Va: Vancomycin.

Considering the situation mentioned, the antagonistic property of four indigenous lactobacilli procured from locally available niches against $K$. pneumoniae has been investigated in order to prepare anti- K. pneumoniae biotherapeutics. The antibacterial activity of non-haemolytic probiotic lactobacilli strains, following agar-well method as well as agar-overlay method, against $K$. pneumoniae has been shown in figure 2 . Among the fermented food products curd belongs to excellent source of probiotic microorganisms, viz. Lactobacillus and being used worldwide dates back to thousands years. Recently, curd has been considered as a novel source of probiotic strains to be used against pathogenic bacteria [2].

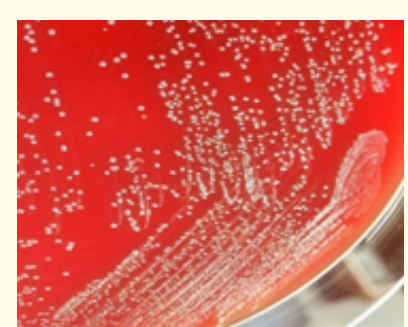

(a) (b)

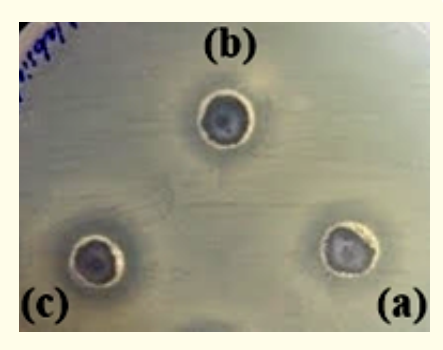

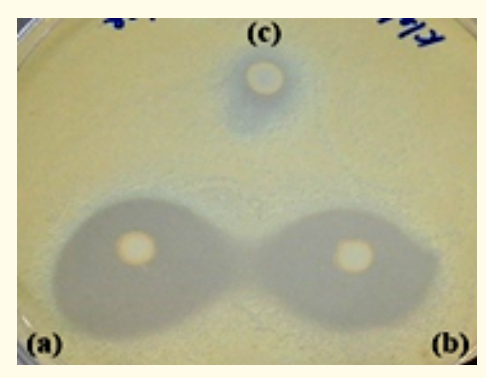

(c)
Figure 2: Lactobacilli strains: (A) $\gamma$-haemolytic colonies of Lactobacillus plantarum LMEM7 strain on sheep blood agar (Hi_Media) plate; (B) Antibacterial activity of lactobacilli (a: $L$. animalis LMEM6; b: L. fermentum MTCC 9748; c: L. acidophilus LMEM8) against $K$. pneumoniae in agar-well method; (C) Antibacterial activity of lactobacilli (a: L. plantarum LMEM 7; b: L. acidophilus LMEM8; c: L. animalis LMEM6) against K. pneumoniae in agar-overlay method.
The antibacterial activity of the test lactobacilli strains against K. pneumoniae, in terms of ZDIs following agar-overlay method, is represented in figure 3; for $K$. pneumoniae B535 clinical strain the ZDIs ranged from $13.00 \pm 1.00 \mathrm{~mm}$ to $33.33 \pm 1.53 \mathrm{~mm}$ (due to the action of L. acidophilus LMEM8 and L. rhamnosus LMEM9, respectively), while the ZDIs ranged from $14.67 \pm 1.53 \mathrm{~mm}$ (from the action of L. acidophilus LMEM8) to $25.33 \pm 1.53 \mathrm{~mm}$ (from the action of L. fermentum MTCC 9748). Following agar-well diffusion method, the ZDIs obtained around the test lactobacilli strains against $K$. pneumoniae ranged from $12.00 \pm 1.00$ to $22.00 \pm 1.00$ (Figure 4). The probiotic bacteria can impede the growth of microbial pathogens within the human gut, utilizing various metabolic pathways, through the synthesis of distinct metabolites, such as hydrogen peroxide, bacteriocin and lactic acid [19-21]. Harith., et al. [8] reported, unlike treatment with aminoglycosides: amikacin and gentamicin, which left live bacterial cells, Lactobacillus supernatant left no live cells in the K. pneumoniae biofilms. Also, the findings, as represented by Lagrafeuille., et al. [7], greatly suggested the lactobacilli culture supernatant as a potent antimicrobial agent against K. pneumoniae. The lactobacilli strains, such as, L. plantarum CIRM653, L. delbrueckii CIRM267 and L. delbrueckii CIRM268, were found effective against K. pneumoniae [22]. The L. fermentum, L. plantarum, L. casei and L. brevis isolated from fermented dairy foods had growth inhibitory activity against $K$. pneumoniae having ZDIs 7 - $14 \mathrm{~mm}$ [23].

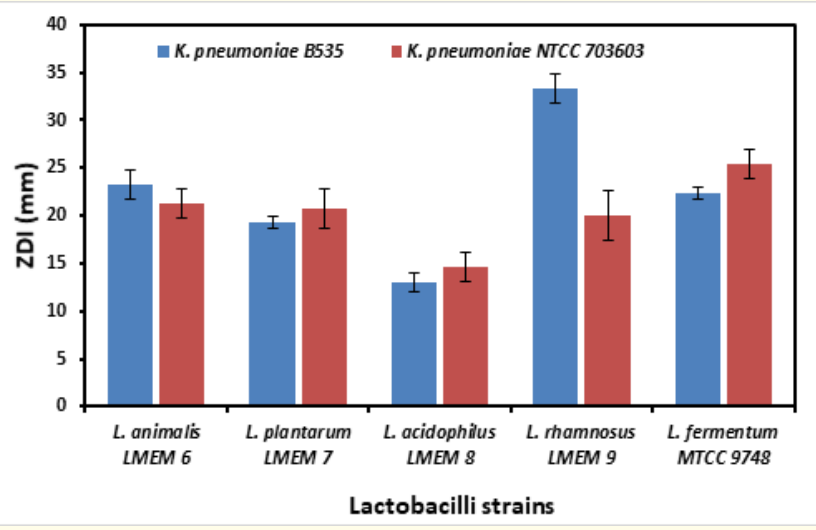

Figure 3: Antibacterial activity of lactobacilli strains in agar-overlay method.

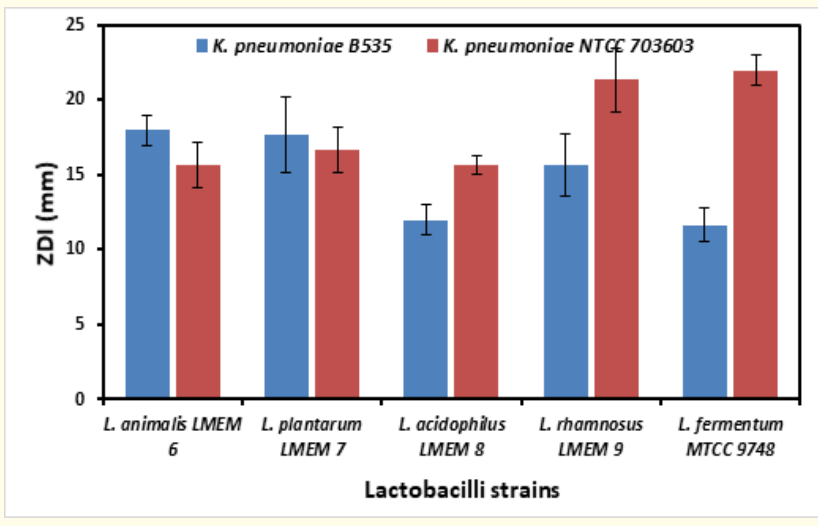

Figure 4: Antibacterial activity of lactobacilli strains in agar-well diffusion method. 
Previously reported that the lactobacilli from curd samples had brilliant antibacterial activity in agar-well diffusion (ZDI: $13.67 \pm$ 0.58 to $29.50 \pm 2.10 \mathrm{~mm}$ ) as well as agar-overlay (ZDI: $11.33 \pm 0.58$ to $35.67 \pm 2.52 \mathrm{~mm}$ ) methods, with "R" values $3.17 \pm 0.29-15.33$ $\pm 1.26 \mathrm{~mm}$ [2]. The "R" values of lactobacilli, in the current study, ranged from 3.00 to 13.17 (Table 2), while the $\mathrm{AU} / \mathrm{mL}$ of the lactobacilli tested against K. pneumoniae ranged from 155.60 to 293.33 (Table 3). The bacteriocin activity has been expressed as $200 \mathrm{AU} /$ ml against Enterococcus faecalis by Kormin., et al [24]. The Lactococcus lactis had strong bacteriocin activity $(1600 \mathrm{AU} / \mathrm{mL})$ against K. pneumoniae ATCC 12296, while some other lactic acid bacteria had inhibitory activity of $800 \mathrm{AU} / \mathrm{mL}$ against Escherichia coli [25]. In our earlier investigation, the average growth inhibitory activity of lactobacilli strains ranged from $233.34 \pm 45.54$ to $280.56 \pm$ 83.67 $\mathrm{AU} / \mathrm{mL}$, against the test bacterial pathogens [2]. Herein, the current study depicts that among the indigenous lactobacilli, L. animalis LMEM6, L. acidophilus LMEM8, L. rhamnosus LMEM9 had high growth inhibition activity, against $K$. pneumoniae, with $\mathrm{R}$ values > $6 \mathrm{~mm}$ (range: 6.5 - 13. $17 \mathrm{~mm}$ ), while, for MTCC strain the R value ranged 7.67 - $9.17 \mathrm{~mm}$; low inhibition capacity was noted for L. acidophilus LMEM8 with "R" values of 3 - 3.84 mm (Table 2). Following agar-overlay method, L. animalis LMEM6, L. rhamnosus LMEM9 and L. fermentum MTCC 9748 strains had strong antagonistic activity against both K. pneumoniae B535 and K. pneumoniae NTCC 703603 indicators strains (Figure 3). The all test lactobacilli including the standard one (L. fermentum MTCC 9748) had high antibacterial activity against K. pneumoniae NTCC 703603 standard strain (ZDI: $\geq$ $15 \mathrm{~mm}$ ), while 3 indigenous lactobacilli strains (L. animalis LMEM6, L. plantarum LMEM7 and L. rhamnosus LMEM9) were found highly active against $K$. pneumoniae $\mathrm{B} 535$ clinical strain (ZDI: $\geq 15 \mathrm{~mm}$ ); $L$. acidophilus LMEM8 and L. fermentum MTCC 9748 strains had moderate activity (ZDI: $11.67 \pm 1.15-12 \pm 1.00 \mathrm{~mm}$ ), following agarwell diffusion method (Figure 4). Thus, this is first to report with scientific validation of anti-K. pneumoniae activity of indigenous curd lactobacilli from this part of the globe.

\begin{tabular}{|l|c|c|c|c|c|}
\hline \multicolumn{1}{|c|}{$\begin{array}{c}\text { Indicator } \\
\text { bacteria }\end{array}$} & \multicolumn{5}{|c|}{ “R” value for lactobacilli strain } \\
\hline & LMEM6 & LMEM7 & LMEM8 & LMEM9 & $\begin{array}{c}\text { MTCC } \\
9748\end{array}$ \\
\hline $\begin{array}{l}\text { K. pneumoniae } \\
\text { B535 }\end{array}$ & 8.17 & 6.17 & 3 & 13.17 & 7.67 \\
\hline $\begin{array}{l}\text { K. pneumoniae } \\
\text { NTCC 703603 }\end{array}$ & 7.17 & 6.84 & 3.84 & 6.5 & 9.17 \\
\hline
\end{tabular}

Table 2: "R" value of lactobacilli isolates against K. pneumonia

\begin{tabular}{|l|c|c|c|c|c|}
\hline \multirow{2}{*}{$\begin{array}{l}\text { Indicator } \\
\text { bacteria }\end{array}$} & \multicolumn{5}{|c|}{$\begin{array}{c}\text { Lactobacilli strain showing growth } \\
\text { inhibitory activity (AU/ml) }\end{array}$} \\
\cline { 2 - 6 } & LMEM6 & LMEM7 & LMEM8 & LMEM9 & $\begin{array}{c}\text { MTCC } \\
9748\end{array}$ \\
\hline $\begin{array}{l}\text { K. pneumoni- } \\
\text { ae B535 }\end{array}$ & 240 & 235.60 & 160 & 208.93 & 155.60 \\
\hline $\begin{array}{l}\text { K. pneumoni- } \\
\text { ae NTCC } \\
703603\end{array}$ & 208.93 & 222.27 & 208.93 & 284.40 & 293.33 \\
\hline
\end{tabular}

Table 3: Growth inhibitory activity of lactobacilli expressed in "AU/ml" for bacterial pathogens

\section{Conclusions}

The study suggests that the indigenous lactobacilli might play an important role in the protection of host against MDR K. pneumoniae infection, and such probiotic strains may beneficially be employed as biotherapeutic agents in partial replacement or adjunct to antibiotic therapy in the treatment of MDR K. pneumoniae infection. Accordingly, the test lactobacilli might be useful in the field of food as well as pharmaceutical industries because of their (lactobacilli) therapeutic potentiality; however, further studies are warranted to extend and authenticate the current indications.

\section{Bibliography}

1. Al-Madboly LA and Abdullah AK. "Potent antagonistic activity of Egyptian Lactobacillus plantarum against multiresistant and virulent food-associated pathogens". Frontiers in Microbiology 6 (2015): 347.

2. Halder D., et al. "Indigenous probiotic lactobacillus isolates presenting antibiotic like activity against human pathogenic bacteria". Biomedicines 5.2 (2017): E31.

3. Paczosa MK., et al. "Klebsiella pneumoniae: going on the offense with a strong defense". Microbiology and Molecular Biology Reviews 80.3 (2016): 629-661.

4. Lebeaux D., et al. "Biofilm-related infections: bridging the gap between clinical management and fundamental aspects of recalcitrance toward antibiotics". Microbiology and Molecular Biology Reviews 78.3 (2014): 510-543.

5. Halder D and Mandal S. "Antibacterial potentiality of commercially available probiotic lactobacilli and curd lactobacilli strains, alone and in combination, against human pathogenic bacteria". Translational Biomedicine 7 (2016): 2.

6. Halder D and Mandal S. "Curd lactobacilli with probiotic potentiality”. Translational Biomedicine 6.2 (2015): 1-6.

7. Lagrafeuille R., et al. "Opposing effect of Lactobacillus on in vitro Klebsiella pneumoniae in biofilm and in an in vivo intestinal colonisation model". Beneficial Microbes 9.1 (2017): 87-100.

8. Al-Mathkhury., et al. "Inhibitory effect of lactobacilli filtrate on Klebsiella pneumoniae Biofilm". The Iraqi Postgraduate Medical Journal 11.1 (2012): 168-179.

9. Clinical and Laboratory Standards Institute (CLSI): Performance standards for antimicrobial susceptibility testing (2011) 21 $1^{\text {st }}$ informational supplement M100S21. CLSI, Wayne, Pa (2011).

10. Nandi S and Mandal S. "Bacteriological profiling of commercially available eye cosmetics and their antibiotic susceptibility pattern". Translational Biomedicine 7.3 (2016): $1-8$.

11. Shokryazdan P., et al. "Probiotic potential of Lactobacillus strains with antimicrobial activity against some human pathogenic strains". BioMed Research International (2014).

12. Carasi P., et al. "Safety characterization and antimicrobial properties of kefir-isolated Lactobacillus kefiri". BioMed Research International (2014): 208974.

13. Pisano MB., et al. "Preliminary evaluation of probiotic properties of Lactobacillus strains isolated from Sardin- 
ian dairy products". BioMed Research International (2014): 286390.

14. Iyapparaj P., et al. "Optimization of bacteriocin production by Lactobacillus sp. MSU3IR against shrimp bacterial pathogens". Aquatic Biosystems 9.1 (2013): 12.

15. Yu WL and Chuang YC. "Clinical features, diagnosis, and treatment of Klebsiella pneumoniae infection" (2018).

16. Lu CH., et al. "Klebsiella pneumoniae meningitis: analysis on clinical features of thirty-two adult patients". Chinese Medical Journal 60.6 (1997): 296-302.

17. Lin WP., et al. "The Antimicrobial Susceptibility of Klebsiella pneumoniae from community settings in Taiwan, a trend analysis". Scientific Reports 6 (2016): 36280

18. Gupta N., et al. "Carbapenem-resistant Enterobacteriaceae: Epidemiology and prevention". Clinical Infectious Diseases 53.1 (2011): 60-67.

19. Collado MC., et al. "The impact of probiotic on gut health". Current Drug Metabolism 10.1 (2009): 68-78.

20. Corr SC., et al. "Understanding the mechanisms by which probiotics inhibit gastrointestinal pathogens". Advance Food and Nutrition Research 56 (2009): 1-15.

21. Sanchez B., et al. "Extracellular proteins secreted by probiotic bacteria as mediators of effects that promote mucosabacteria interactions". Microbiology 156.11 (2010) 32323242.

22. Vuotto C., et al. "Probiotics to counteract biofilm-associated infections: promising and conflicting data". International Journal of Oral Science 6.4 (2014) 189-194.

23. Saranya S and Hemashenpagam N. "Antagonistic activity and antibiotic sensitivity of Lactic acid bacteria from fermented dairy products". Advances in Applied Science Research 2.4 (2011): 528-534.

24. Kormin S., et al. "Bacteriocin-producing lactic acid bacteria isolated from traditional fermented food". Malaysian Journal of Medical Sciences 8.1(2001): 63-68.

25. Shehata MG., et al. "Screening of isolated potential probiotic lactic acid bacteria for cholesterol lowering property and bile salt hydrolase activity". Annals of Agricultural Sciences 61.1 (2016): 65-75.

Volume 1 Issue 4 April 2018

(C) All rights are reserved by Shyamapada Mandal and

Debashis Halder. 